\title{
Review of: "Association of sleep duration in middle and old age with incidence of dementia"
}

\author{
Jian $\mathrm{Liu}^{1}$ \\ 1 Brock University
}

Potential competing interests: The author(s) declared that no potential competing interests exist.

Review of "Association of sleep duration in middle and old age with incidence of dementia"

This is a study of examination of the association between sleep duration and risk of dementia among 7,959 participants from the Whitehall II study ${ }^{1}$. The main finding of this study is that people with a short sleep duration in midlife are at an increased risk of dementia in late life. Compared to previous studies, this study apparently has two unique features:

1. This study started with participants at age 50 and had 25 years average follow-up time, which provided a unique opportunity to evaluate the long impact of sleep duration in midlife on dementia risk in later life. Among previous cohort studies that focus on the relationship between sleep duration and risk of dementia, most of them had follow-up time less than 10 years. However, a chronic disease like dementia that takes long time to be diagnosed with its pathophysiological changes over time gradually, a follow-up time shorter than 20 years may not be able to provide a comprehensive picture on the impact of sleep duration on the risk of dementia.

2. The analytical strategies used in this study have well addressed the research question: a) with the entire sample, it examined the risk association of dementia with a short sleep duration ( $f 6 \mathrm{~h}$ ) with the sleep duration information reported at age 50,60 , and $70 ; b)$ with a sub sample ( $n=6875$ ), in which participants provided at least two out of the three sleep duration information at age 50,60, and 70, it examined whether the trajectory of persistent short sleep duration ( $₫ 6 \mathrm{~h}$ ) identified at the three points of age increases the risk of dementia; c) furthermore, it used accelerometer-assessed sleep duration, an objective measurement of sleep duration, collected among a sub-sample $(n=4267)$ and examined the risk association of dementia with a short sleep duration. The results from the three approaches all suggested that a short sleep duration in midlife increased the risk of dementia in later life (though this risk association was not statistically significant at age 70 when analysis was conducted with the entire sample), after controlling for social demographic, behavioural, cardiometabolic, and mental health factors.

The results of this study are interesting and very clearly organized and presented. However, like any other works, there always are some rooms for improvement. Here are some comments for authors' 
consideration:

1. This study only identified the risk association on short sleep duration with dementia, and not like other similar studies, with significant findings only for a long sleep duration at the baseline or the trajectory of change to long sleep duration during the follow-up increasing dementia risk ${ }^{2} 3$, or a U-shape risk association of sleep duration with dementia ${ }^{4}$. The authors noted these differences, but it would be grateful if they could have given a more thoughtful discussion. For instance, the definition for what is considered as short or long sleep duration in these studies was in fact not consistence; the definition of short sleep duration could vary from $£ 5 \mathrm{~h}$ to $<7 \mathrm{~h}$; while long sleep duration could be from $>8 \mathrm{~h}$ to $\geq$ $10 \mathrm{~h}$. These sleep durations could refer it as either within 24-h time frame or just nighttime ${ }^{2}$.

2. The results of this study had demonstrated that the identified risk association between short sleep duration and dementia was independent of some known social demographic, cardiometabolic, behavioural, and mental health factors. Although the authors admitted that as the observational nature, this study cannot preclude residual confounding effect, it is worth of discussion of some other known factors that may play a role on the risk of dementia. For instance, high levels of lipids ${ }^{5}$, inflammation ${ }^{6}$, and night shift work or long working hour ${ }^{7}$. Particularly, when the Whitehall II study might have such information being collected.

3. The results of this study lead to a conclusion that short sleep duration in midlife is associated with an increased risk of late-onset dementia. Authors gave a very general brief on the association between sleep and cognitive function, but unfortunately, they did not try to further explore what might be the potential mechanism or rationales on what they have observed, ie, why does short sleep duration in midlife increase the dementia risk in late life? Is sleep duration related to the process of the management of waste products in brain? We don't know, it might be ${ }^{8}$. Since our brains consume at least $20 \%$ daily energy, certainly it needs time to maintain its normal status such as cleaning waste products and information process, and thus, too short sleep duration may not meet its needs.

4. Some citated references did not match with the contents. For instance, in the third paragraph of the background introduction "In our analyses, we examine whether mental disorders in midlife affects the association of sleep duration with dementia. Given potential bias in self-reported measures of sleep duration, we examine the association between objectively assessed sleep duration and risk of dementia in a sub-sample 20 of the study." the reference citation 20 is "Silverberg, N., Elliott, C., Ryan, L., Masliah, E. \& Hodes, R. NIA commentary on the NIA-AA Research Framework: Towards a biological definition of Alzheimer's disease. Alzheimers Dement 14, 576-578 (2018)." It seems not to be what the authors referred to. Also in the discussion, the statement that "Two recent meta-analyses suggest a U-shaped association between sleep duration and incident dementia, with lower risk in people sleeping $7 \mathrm{~h}$ per night, and greater risk among those with shorter sleep and also among those with longer sleep duration 4,5. "may be misleading. Only citation 4 “Liang, Y., Qu, L. B. \& Liu, H. Non-linear associations between sleep duration and the risks of mild cognitive impairment/dementia and cognitive decline: a dose-response meta-analysis of observational studies. Aging Clin. Exp. Res 31, 309-320 (2019)." 
indicated a non-linear association or a U-shape association, but the study in citation 5 “Fan, L., Xu, W., Cai, Y., Hu, Y. \& Wu, C. Sleep Duration and the Risk of Dementia: A Systematic Review and Metaanalysis of Prospective Cohort Studies. J. Am. Med Dir. Assoc. 20, 1480-1487 e1485 (2019)." Actually indicated that the increased risk of dementia was only identified for a long sleep duration.

1. Sabia S, Fayosse A, Dumurgier J, et al. Association of sleep duration in middle and old age with incidence of dementia. Nat Commun 2021;12(1):2289. doi: 10.1038/s41467-021-22354-2

2. Fan L, Xu W, Cai Y, et al. Sleep Duration and the Risk of Dementia: A Systematic Review and Metaanalysis of Prospective Cohort Studies. J Am Med Dir Assoc 2019;20(12):1480-87 e5. doi:

10.1016/j.jamda.2019.06.009

3. Lu Y, Sugawara Y, Zhang S, et al. Changes in sleep duration and the risk of incident dementia in the elderly Japanese: the Ohsaki Cohort 2006 Study. Sleep 2018;41(10) doi: 10.1093/sleep/zsy143

4. Liang Y, Qu LB, Liu H. Non-linear associations between sleep duration and the risks of mild cognitive impairment/dementia and cognitive decline: a dose-response meta-analysis of observational studies. Aging Clin Exp Res 2019;31(3):309-20. doi: 10.1007/s40520-018-1005-y

5. Schilling S, Tzourio C, Soumare A, et al. Differential associations of plasma lipids with incident dementia and dementia subtypes in the $3 C$ Study: A longitudinal, population-based prospective cohort study. PLoS Med 2017;14(3):e1002265. doi: 10.1371/journal.pmed.1002265

6. Grande G, Marengoni A, Vetrano DL, et al. Multimorbidity burden and dementia risk in older adults: The role of inflammation and genetics. Alzheimers Dement 2021;17(5):768-76. doi: 10.1002/alz.12237

7. Nabe-Nielsen K, Hansen AM, Ishtiak-Ahmed K, et al. Night shift work, long working hours and dementia: a longitudinal study of the Danish Work Environment Cohort Study. BMJ Open 2019;9(5):e027027. doi: 10.1136/bmjopen-2018-027027

8. Komaroff AL. Does Sleep Flush Wastes From the Brain?JAMA 2021 doi: 10.1001/jama.2021.5631 